\title{
Preparation of Cross-sectional Samples of Proton Exchange Membrane Fuel Cells for TEM Characterization
}

\author{
D. A. Blom* and J. R. Dunlap** \\ *Oak Ridge National Laboratory, Oak Ridge, Tennessee 37831-6064 \\ **Program in Microscopy, The University of Tennessee, Knoxville, TN 37996
}

Proton exchange membrane (PEM) fuel cells are a possible low-emission power source for vehicles in the future. The U.S. Department of Energy has announced both the FreedomCAR and FreedomFuel programs with the stated intention of moving the United States towards a hydrogen-based economy and away from dependence on imported crude oil for America's energy needs. Currently, the cells require large quantities of expensive precious metal catalysts to work efficiently. Additionally, the performance of the cells degrades over time. TEM characterization of PEM fuel cells before and after electrochemical aging may provide information about optimal quantities and distribution of precious metal catalysts, and the microstructural/microchemical basis of the degradation upon use. The initial challenge for characterization of PEM fuel cells is sample preparation.

The electrodes of PEM fuel cells consist of a gas permeable mixture of supported catalyst particles, proton conducting ionomer, electron conducting carbon cloth and hydrophobic material. The electrodes are typically hot-pressed with a proton conductive polymer such as Nafion. The changes in both the polymer membrane and the electrode structure are of interest. Previous investigations of fuel cells have either looked at only one component of the system such as the electrocatalyst alone, or have removed only part of the cell for observation [1]. Given a desire for a large uniform thin area over all of the cell constituents, and the porosity of the electrodes, ultramicrotomy was investigated as a suitable sample preparation technique [2].

Samples of a PEM fuel cell were embedded in Araldite 6005, and cured at $60^{\circ} \mathrm{C}$ overnight in air. Thin cross sections of the PEM fuel cell in the Araldite epoxy were produced with either a Reichert OMU3 or Leica UCT ultramicrotome at room temperature. A $45^{\circ}$ diamond knife was used for sectioning. The samples were collected on 200-300 mesh copper grids for TEM examination. Both an Hitachi HF2000 field emission TEM and an Hitachi HD-2000 field emission STEM were used to characterize the PEM fuel cells. Figure 1 is a montage of low magnification micrographs taken on the Hitachi HF-2000 illustrating how ultramicrotomy successfully produced large-area thin sections of specimens that have a number of components with widely varying mechanical properties. In Figure 2 a Z-contrast STEM image of a nominally $50 \mathrm{~nm}$ thick section of a PEM fuel cell is shown. The small bright features are the Pt-based catalyst particles. Both the porosity of the electrode and the spatial and size distribution of the catalyst are clearly seen in the micrograph. Figure 3 shows the interface between the PEM and electrode of a fuel cell that had been tested for 325 hours. EDS analysis showed that the interface between the PEM and the electrode was enriched in $\mathrm{S}$ and depleted in $\mathrm{F}$ with respect to an unaged cell.

We conclude that room temperature diamond knife ultramicrotomy can successfully produce crosssectional samples of PEM fuel cells. Work in ongoing to understand the aging mechanisms for PEM fuel cells.

\section{References}

[1] E. A. Ticianelli et al., J. Appl. Electrochem., 21(1991)(7) 597. 
[2] T. F. Malis and D. Steele, in R. Anderson, editor, Specimen Preparation for Transmission Electron Microscopy of Materials II, vol. 199 of Materials Research Society Symposium Proceedings, pages 3-42, Materials Research Society, 1990.

[3] Research sponsored by the U.S. Department of Energy, Assistant Secretary for Energy Efficiency and Renewable Energy, Office of Hydrogen, Fuel Cells, and Infrastructure Technologies Program under contract DE-AC05-00OR22725 with UT-Battelle, LLC.

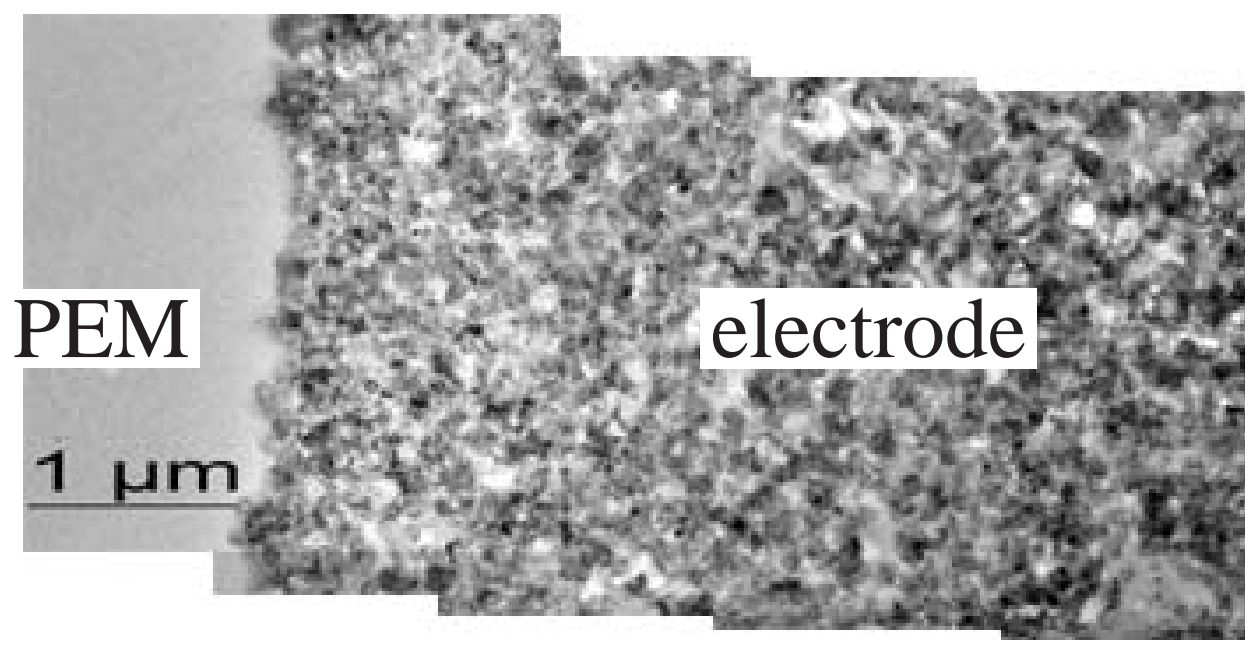

Figure 1: Montage of micrographs illustrating the ability of ultramicrotomy to produce large, uniform thin areas.

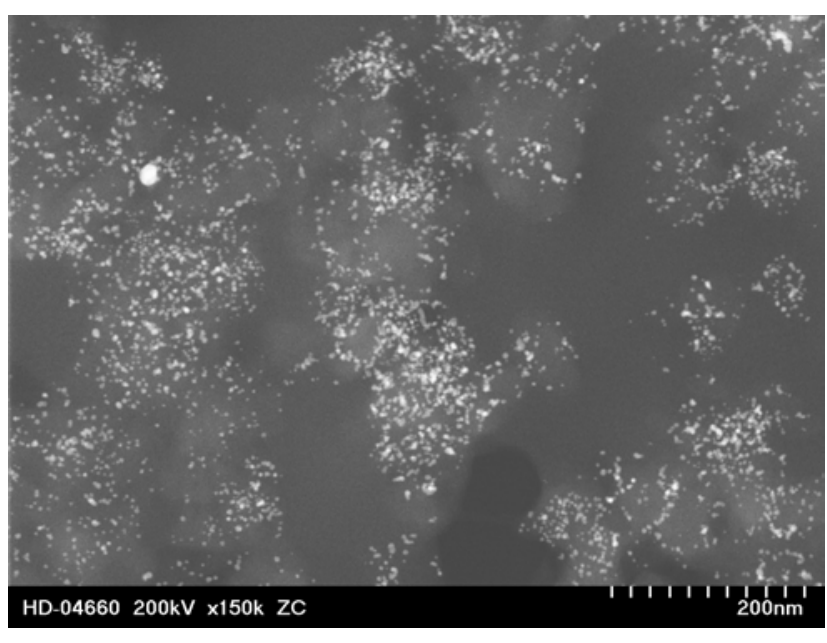

Figure 2: Z-contrast image of a $50 \mathrm{~nm}$ thick section of PEM fuel cell. The inindividual catalyst particles and arrangement of the various components is visible in this image.

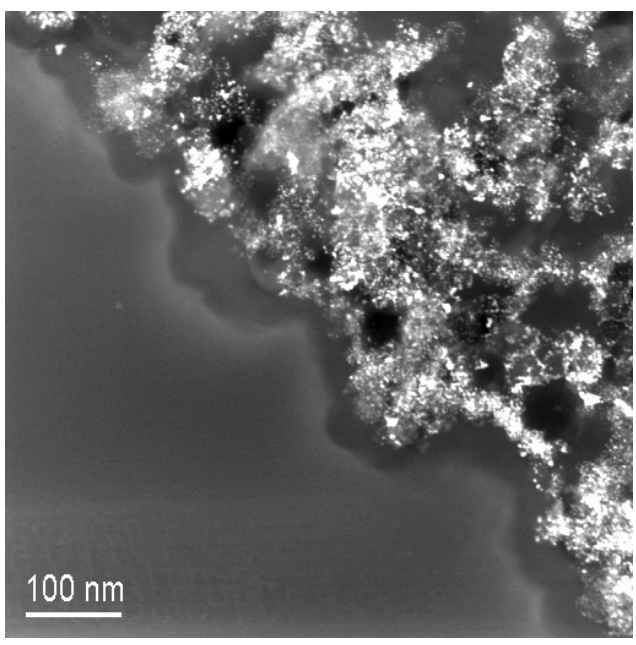

Figure 3: Z-contrast image of the PEM/electrode interface in an aged fuel cell. EDS analysis showed the interface is enriched in $\mathrm{S}$ and depleted in $\mathrm{F}$ relative to an unaged cell. 